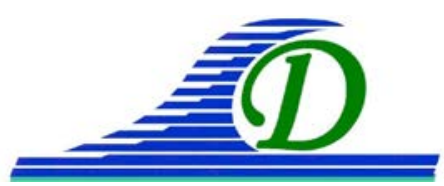

XIII ${ }^{e ̀ m e s}$ Journées Nationales Génie Côtier - Génie Civil

Dunkerque, 2-4 juillet 2014

DOI:10.5150/jngcgc.2014.047 C Editions Paralia CFL

disponible en ligne - http://www.paralia.fr - available online

\title{
Propagation de crue et aléa de submersion. Étude de cas : l’Oued Sebou - Kénitra (Maroc)
}

\author{
Julie LEBUNETEL ${ }^{1}$, Pierre FARNOLE ${ }^{1}$, Khalid LAZRAK $^{2}$
}

1. ERAMM NANTES, 15 rue Crébillon, 44000 Nantes, France.

jl@eramm.fr ; pf@eramm.fr

2. MDC Ingénierie, Résidence Ibn Sina, 10106 Agdal, Maroc.

klazrakbis2@gmail.com

\section{Résumé :}

L'étude hydrodynamique du tronçon du bas Sebou, compris entre l'embouchure de l'oued et le pont Oulad Berjal, s'inscrit dans le cadre d'une réflexion menée actuellement par la direction régionale de l'équipement et des transports de Kénitra sur les risques d'inondation de la ville de Kénitra et les possibilités d'aménagement des deux rives de l'oued Sebou pour sécuriser les terrains aménagés. Le débit de l'Oued Sebou est contrôlé par un nombre important de barrages en amont du pont Oulad Berjal. Cependant, sous certaines conditions de crues, de marnage et de houle à l'embouchure, l'oued peut déborder de son lit comme cela s'est produit en 2010 (entre le 15 et le 28 février). Le débit de pointe mesuré au pont atteint les $3500 \mathrm{~m}^{3} / \mathrm{s}$ avec un marnage qui peut atteindre $3.4 \mathrm{~m}$ en condition de vive eau et une houle sévère entrant dans le chenal $(\mathrm{Hs}=4,5 \mathrm{~m})$ limitant l'évacuation des crues.

Notre travail a consisté à reproduire les phénomènes observés, à déterminer l’influence du marnage et de la houle sur l'écoulement des crues et ses conséquences sur les zones inondables. Une campagne de mesures de la marée, du courant et de la houle sur 2 mois a permis le calage du modèle numérique (REFLUX) qui a été mis en œuvre. Les forçages de débit, vent, marnage et houle (via un couplage avec le logiciel REFONDE) ont été conjointement appliqués. Le domaine est étendu et les conditions hydrodynamiques sévères. Les temps de calcul sont longs mais la mise en œuvre de REFLUX a permis de définir les cartes d'aléa de submersion de l'état actuel. Dans un deuxième temps, les zones de protection et les variantes d'aménagement pour la protection sont définies pour permettre la mise en place d'une stratégie de développement et d'aménagement du secteur concertée et durable.

Mots-clés : Crues, Aléa de submersion, Hydrodynamique, Modélisations numériques, Agitation côtière, Maroc.

\section{Introduction}

Kénitra se situe sur la façade Atlantique du Maroc, à environ $200 \mathrm{~km}$ au sud de Tanger. Le bassin de l'oued Sebou est parmi les plus arrosés du Maroc. Son bassin totalise une superficie d'environ $40000 \mathrm{~km}^{2}$. Le bassin du Sebou produit près du tiers des eaux de 


\section{Thème 2 - Dynamique sédimentaire}

surface du Maroc. Le bassin de Sebou est doté de plusieurs aménagements hydrauliques qui, bien que destinés à des fins telles que la régularisation des eaux pour l'alimentation en eau potable et l'irrigation ou la production d'énergie, jouent un rôle important dans le laminage des crues. L'influence des marées sur le fonctionnement hydrologique de l'estuaire du Sebou est démontrée avec la caractérisation de deux masses d'eau qui s'opposent et un remplissage de l'estuaire par le fond plus rapide que la vidange (FEKHAOUI \& EL BLIDI, 2003).

La plaine du Gharb, impactée notamment par l'Oued Sebou, a connu de sévères inondations (1963, 1973, 1989, 1996, 2002, 2008, 2009 et 2010). Ces événements destructeurs ont provoqué l'inondation de plusieurs milliers d'hectares, occasionnant ainsi d'importants dommages économiques et sociaux (ABHS, 2008).

Sur la façade maritime, l'embouchure subit de fortes contraintes d'agitation. L'embouchure est endiguée et les épis ainsi créés font partiellement obstacle au transit sédimentaire. Cependant, une partie du transit littoral se dépose au niveau de l'embouchure de l'oued (plage de Mehdia) (SOGREAH \& LPEE, 2011. Depuis huit ans, $500000 \mathrm{~m}^{3}$ en moyenne de sédiments sont dragués chaque année (principalement du sable). La crue exceptionnelle de 2010 de l'oued Sebou a entraîné des apports solides de l'ordre de $800000 \mathrm{~m}^{3}$, ce qui a eu pour conséquence le blocage de la navigation et une fermeture de 6 mois du port de Kénitra.

Il s'agit donc d'un secteur où le contexte hydrodynamique et sédimentaire est complexe, subissant l'influence de l'Oued, du marnage et de l'agitation se propageant dans le chenal. Il convient ainsi, préalablement à l'évaluation des possibilités d'aménagement et d'exploitation du territoire, de définir l'aléa de submersion associé à un évènement exceptionnel.

\section{Les conditions naturelles}

L'analyse de l'état actuel a été réalisée dans le cadre de cette étude, notamment à partir d'une large campagne de mesures (topo-bathymétrie, houle, courants, hauteurs d'eau, climatologie, sédimentologie). Celle-ci a permis d'établir les conditions initiales ainsi que les conditions hydrodynamiques représentatives du secteur d'étude pour la réalisation des modélisations numériques.

Plusieurs points de mesure ont été mis en place dans l'oued pour la mesure des courants (sur l'ensemble de la colonne d'eau) et des hauteurs d'eau (voir figure 1). On constate l'influence de la marée sur l'écoulement de l'Oued avec propagation de l'onde de marée jusqu'au pont (20km de l'embouchure).

Les houles les plus fréquentes proviennent des secteurs ONO à NW, soit l'orientation de l'embouchure de l'Oued.

Le climat régnant sur l'ensemble du bassin est de type méditerranéen à influence océanique. On distingue une saison humide, d'octobre à mai, qui reçoit environ $90 \%$ des précipitations totales annuelles, et une saison sèche, de juin à septembre. 


\section{XIII ${ }^{\text {èmes }}$ Journées Nationales Génie Côtier - Génie Civil \\ Dunkerque, 2-4 juillet 2014}

Le niveau moyen de la mer dans la région de Kenitra/Rabat est voisin de +2,17 mCM. Sur le littoral atlantique marocain, la marée est de type semi-diurne avec une période de 12h25min. Le marnage d'une marée de vive-eau exceptionnelle (VEE) est de $3.40 \mathrm{~m}$.

On tient compte par ailleurs des surcotes de tempêtes et du niveau pessimiste d'élévation de la mer dû au changement climatique à l'horizon 2100 (DGEC, 2010).

Les conditions testées sont présentées dans le tableau 1.

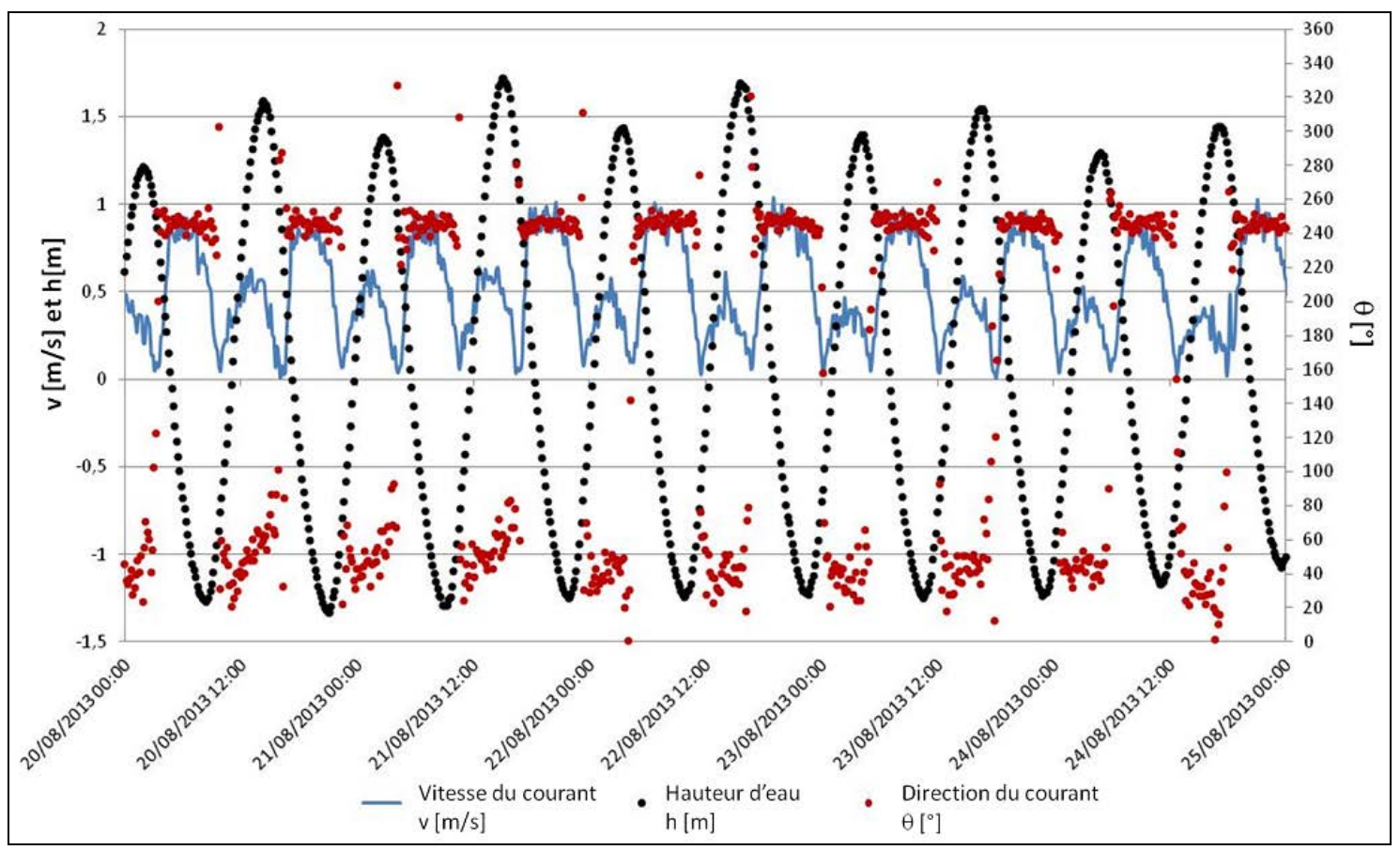

Figure 1. Exemple de hauteurs d'eau et courants mesurés en surface à $8 \mathrm{~km}$ en amont de l'embouchure.

Tableau 1. Conditions testées.

\begin{tabular}{|c|c|c|c|c|c|c|c|c|}
\hline & \multicolumn{3}{|c|}{ Houle au large } & \multicolumn{2}{|l|}{ Vent } & \multicolumn{2}{|c|}{ Niveau d'eau } & \multirow{2}{*}{$\begin{array}{l}\text { Débits } \\
Q\left[\mathrm{~m}^{3} / \mathrm{s}\right]\end{array}$} \\
\hline & $H s[m]$ & $T p[s]$ & $\theta\left[{ }^{\circ} \mathrm{N}\right]$ & $u[m / s]$ & $\theta\left[{ }^{\circ} N\right]$ & Marée & $\begin{array}{l}\text { CI Cote d'eau } \\
\text { [mCM] }\end{array}$ & \\
\hline Calage & 0.86 & 9.12 & 305.7 & 2 & 4 & $M E$ & 2.93 & 350 \\
\hline \multirow{3}{*}{$\begin{array}{l}\text { Climat } \\
\text { moyen }\end{array}$} & 1.96 & 11 & 311 & 5 & 270 & $V E E$ & 4.03 & QJ \\
\hline & 1.96 & 11 & 311 & 5 & 270 & $M M$ & 3.03 & $Q J$ \\
\hline & 1.96 & 11 & 311 & 5 & 270 & $M E$ & 2.93 & $Q J$ \\
\hline \multirow{4}{*}{$\begin{array}{l}\text { Climat } \\
\text { sévère }\end{array}$} & 4.4 & 18 & 320 & 7 & 270 & $V E E$ & 4.03 & $Q I X=3500$ \\
\hline & 4.7 & 19.5 & 320 & 7 & 270 & $V E E$ & 4.03 & $Q I X=3500$ \\
\hline & 5 & 19.5 & 320 & 7 & 270 & $V E E$ & 4.03 & $Q I X=3500$ \\
\hline & 4.5 & 15 & 305 & 7 & 270 & $V E E$ & 4.03 & $Q I X=3500$ \\
\hline
\end{tabular}

Les données topo-bathymétriques les plus récentes disponibles ont été utilisées dont le lever depuis le pont (voir figure 2) jusqu’à l'embouchure réalisé en 2013. 


\section{Modélisations numériques}

L'agitation, la courantologie et les hauteurs d'eau associées aux conditions de climat moyen et de climat sévère ont été analysées après calage du modèle vis-à-vis du climat le plus probable mesuré au cours de la campagne de mesures.

\subsection{L’agitation}

Un modèle de propagation (SWAN) a été réalisé afin de déterminer les conditions d'entrée du modèle d'agitation. Le modèle d'agitation à la côte (REFONDE) a ensuite permis de déterminer l'impact des houles dans l'embouchure. Les principaux résultats sont :

a) les ouvrages ont un impact important sur la propagation de la houle dans le chenal, avec des phénomènes de réflexion significatifs ;

b) la houle de secteur NW pénètre directement dans le chenal et les digues de l'oued servent de guide d'onde jusqu'à la conserverie ;

c) l'agitation diminue brutalement au droit de la conserverie (voir figure 2) et n'atteint pas le port de Mehdia, même pour des conditions centennales.

\subsection{Courants et hauteurs d'eau}

\subsubsection{Calage du modèle}

Le calage a été réalisé avec la configuration morphologique initiale du domaine. Un événement représentatif de la situation hydrodynamique de l'estuaire au cours de la période de mesure a été sélectionné. Les caractéristiques de l'évènement ont été mesurées au cours de la campagne de mesure en plusieurs points le long de l'Oued (courant, hauteur d'eau) et à l'embouchure (houle, vent...). Les caractéristiques de houle de cet évènement sont : $H s=0.9 \mathrm{~m}, T p=9.1 \mathrm{~s}, \theta=305.7^{\circ} \mathrm{N}$. Le vent au port de Mehdia était alors d'une intensité de $2 \mathrm{~m} / \mathrm{s}$ et de provenance $4^{\circ} \mathrm{N}$. Viscosité et rugosité numériques ont été fixées de manière à reproduire numériquement l’évènement.

\subsubsection{Climat moyen - influence du marnage}

Les modélisations de courantologie en climat moyen n’ont pas montré de débordements. L'impact du marnage dans ces conditions a pu être observé. Pour les conditions testées, les courants sont toujours sortants mais au cours du flot, ils décroissent fortement en particulier au niveau de l'embouchure où l'écoulement de marée s’oppose à l'écoulement de l’oued (voir figure 2). 


\section{XIII ${ }^{\text {èmes }}$ Journées Nationales Génie Côtier - Génie Civil \\ Dunkerque, 2-4 juillet 2014}

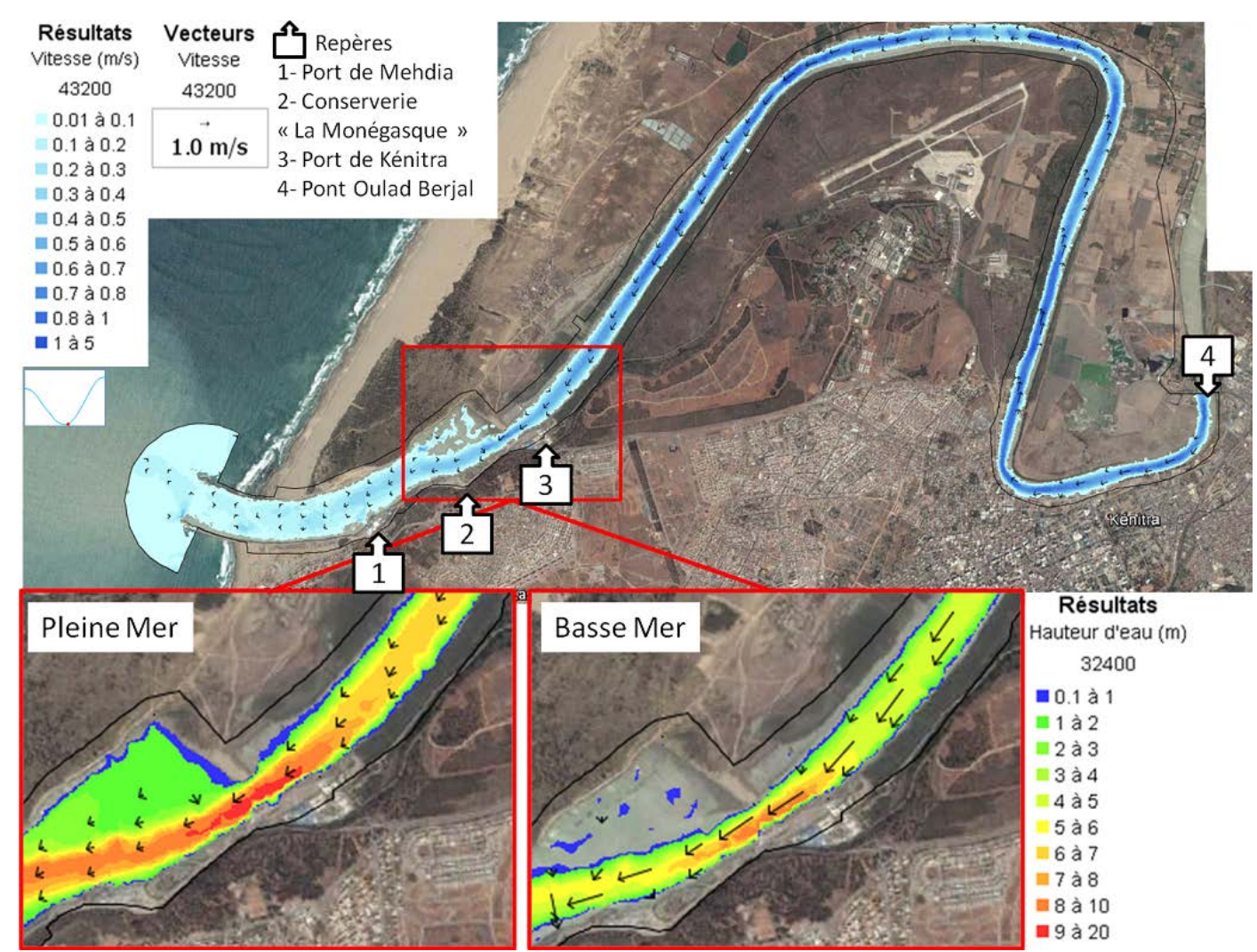

Figure 2. Courants et hauteurs d'eau - Climat moyen (Image aérienne Google Earth V7).

Les marées de mortes eaux et les marées moyennes montrent des résultats très proches. Cependant, les marées de vives eaux montrent des variations de vitesses et de hauteurs d'eau plus importantes. En condition d'écoulement moyen journalier de l'oued Sebou, l'influence du marnage est significative à l'embouchure et diminue à l'amont jusqu'à être quasi inexistante au pont, limite du modèle.

\subsubsection{Climat sévère - expansion des crues}

Considérant l'expansion des crues, l'étude s'est concentrée sur l'hydrogramme de crue de 2010 (voir figure 3). Une marée de vive eau a été sélectionnée ainsi qu'un fort vent et des conditions de houle fortes avec différentes périodes de retour. Quelle que soit la condition de houle testée, les modélisations de courantologie n’ont pas montré d'impact significatif sur l'évacuation de la crue de 2010.

La propagation de la crue n'a pas montré d'inondations aux abords de l'embouchure jusqu’à la zone portuaire de Kénitra. En rive droite et $1 \mathrm{~km}$ en amont du port de Kénitra, il existe au maximum de l'expansion de la crue, un secteur inondé le long de l'oued sur environ $1.5 \mathrm{~km}$ mais celui-ci est restreint (n'atteint pas la route) et ne dépasse pas l'aléa faible. Plus en amont, l'oued sort de son lit sur la rive gauche le long du terrain militaire. Les zones les plus impactées par la crue se situent entre le terrain militaire et le pont, rive droite. Les premières inondations se situent sur la rive droite 


\section{Thème 2 - Dynamique sédimentaire}

immédiatement à proximité du pont. Les eaux se propagent ensuite vers la zone de dépression topométrique. Des débordements en rive gauche se produisent aussi au débit de pointe de la crue (voir figure 4).

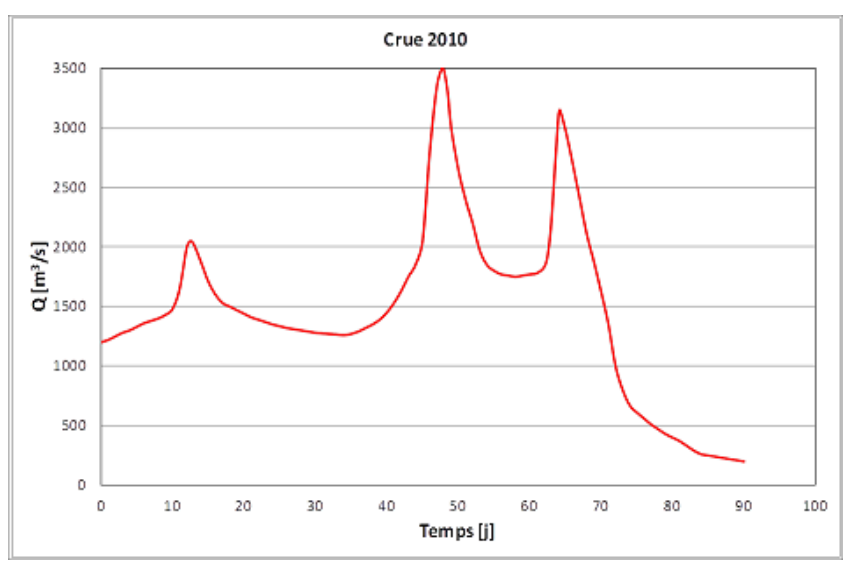

Figure 3. Hydrogramme de crue de l'Oued Sebou de janvier à mars 2010.

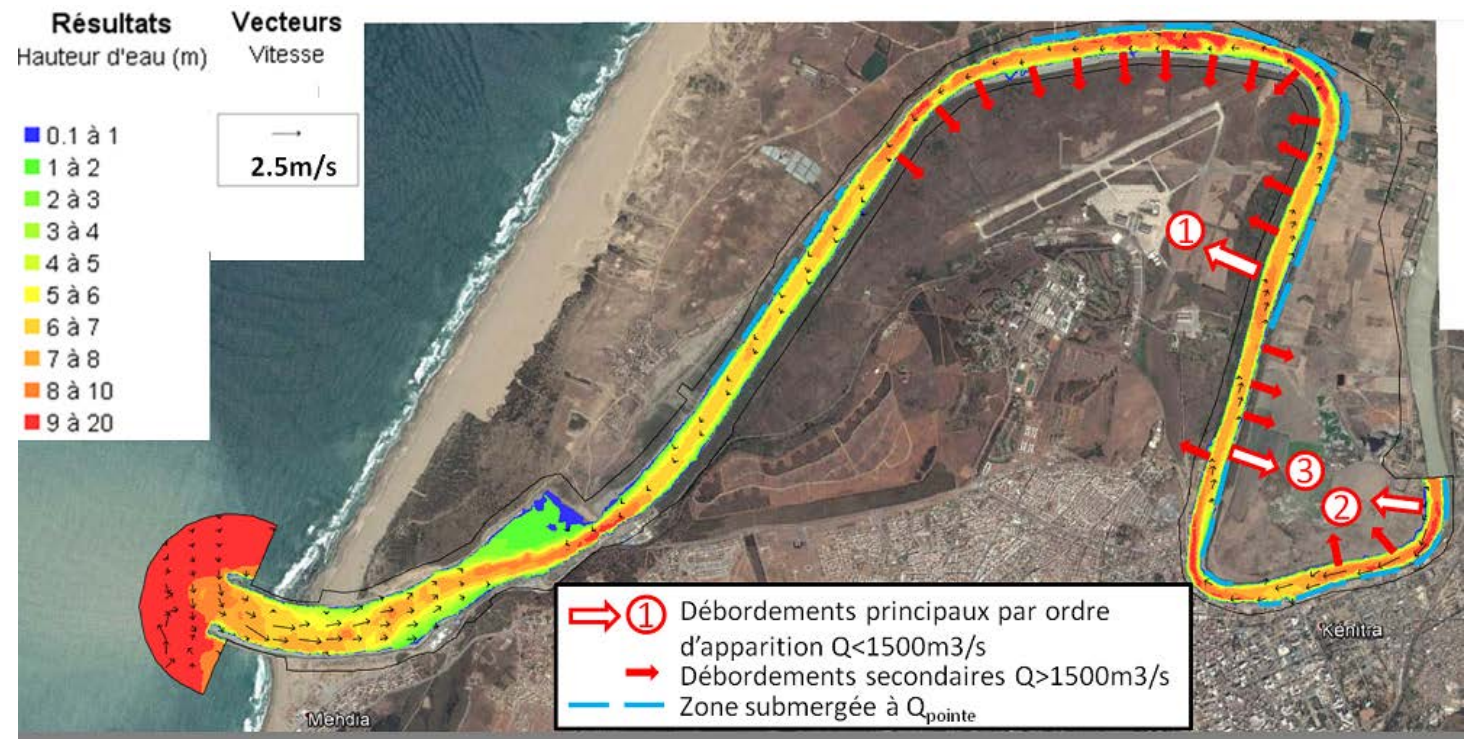

Figure 4. Analyse des débordements constatés au cours de la crue (Image aérienne Google Earth V7).

\section{Cartes d'aléa}

Dans cette partie ont été analysés les résultats simultanés de courants et hauteurs d'eau. Ceux-ci ont permis d'établir les cartes d'aléa de submersion. La figure 5 présente la carte d'aléa de submersion associée au débit de pointe de la crue de 2010. Trois catégories d'aléa d'inondation sont définies :

- aléa faible $(u<0.5 \mathrm{~m} / \mathrm{s}$ et $h<0.5 \mathrm{~m})$ présenté en vert ;

- aléa modéré $(0.5<u<1 \mathrm{~m} / \mathrm{s}$ et $0.5<h<1 \mathrm{~m})$ présenté en jaune ;

- aléa fort ( $u>1 \mathrm{~m} / \mathrm{s}$ et $h>1 \mathrm{~m})$ présenté en rouge. 


\section{XIII ${ }^{\text {èmes }}$ Journées Nationales Génie Côtier - Génie Civil \\ Dunkerque, 2-4 juillet 2014}

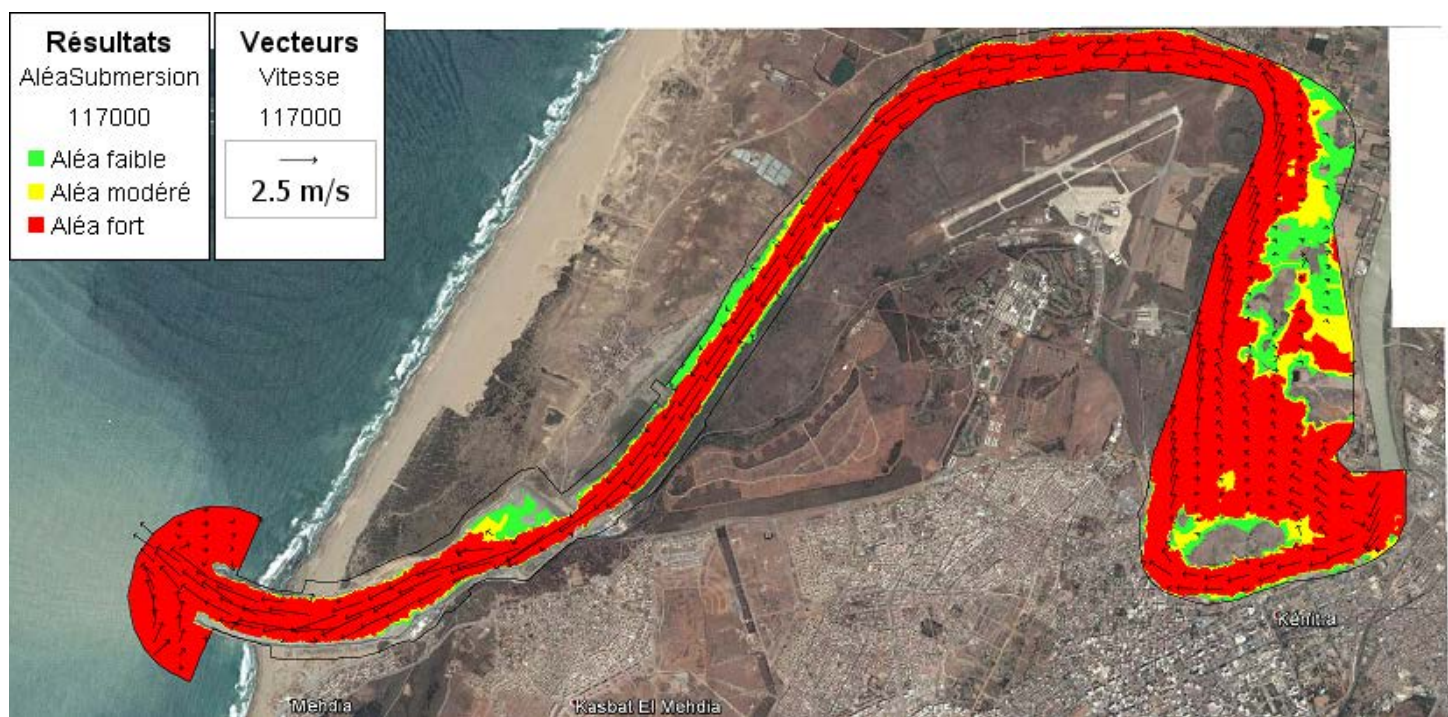

Figure 5. Carte d'aléa de submersion de la crue (Image aérienne Google earth V7).

A partir des cartes d'aléa et des profils transversaux, on détermine à $10 \mathrm{~m}$ la cote minimale des berges à fixer lors de l'aménagement de la zone pour réduire l'aléa de submersion associé à la crue de 2010 de débit de pointe $3500 \mathrm{~m} 3$.

Au total cela représente $2700 \mathrm{ml}$ en rive droite et $1500 \mathrm{ml}$ en rive gauche. Les berges rive gauche sont cependant légèrement plus hautes $(8-10 \mathrm{~m})$ que celles rives droite (6-8 $\mathrm{m})$ et ne nécessitent pas les mêmes installations. Ces éléments sont une première estimation qui permet tout de même de définir les secteurs à risques. Les résultats permettent de localiser les secteurs qui nécessitent un aménagement avant tout développement (voir figure 6).

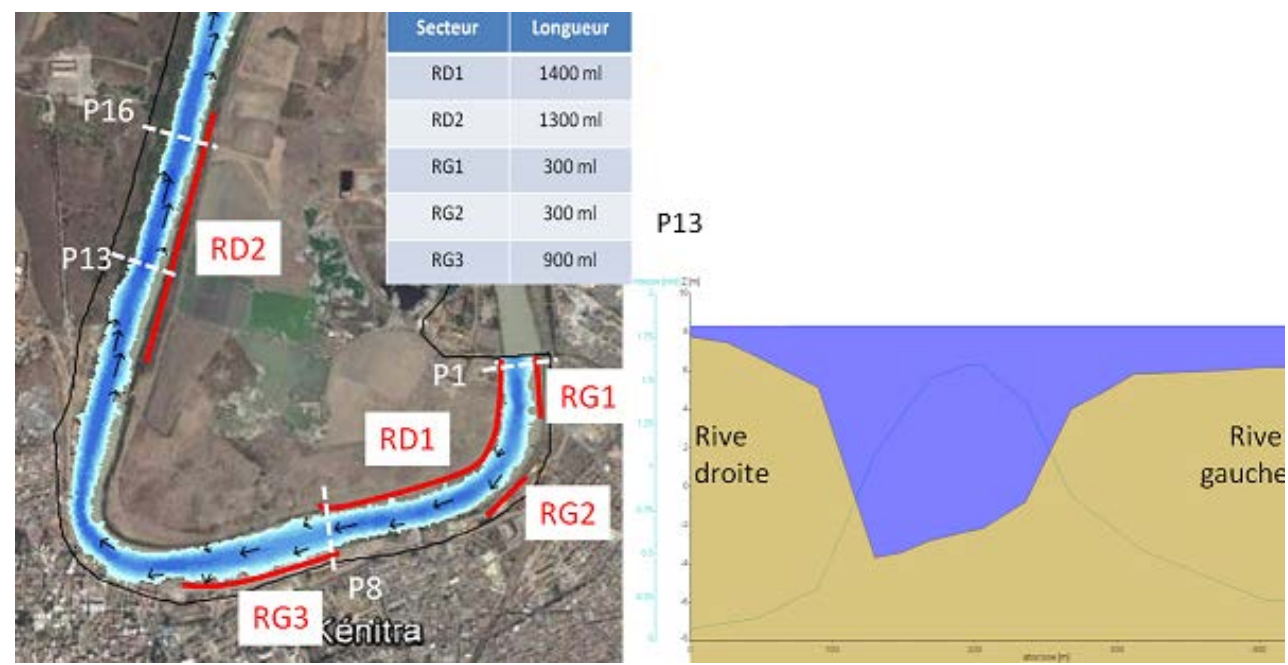

Figure 6. Profils et linéaire à traiter prioritairement (Image aérienne Google Earth V7). 


\section{Conclusion}

L'étude bibliographique et les mesures de terrain réalisées dans le cadre de ce projet ont permis de mettre en œuvre une chaîne de modélisations adaptée pour l'étude de submersion de l'Oued Sebou.

Rive gauche, des aménagements sont nécessaires pour protéger la ville de Kénitra des inondations, avec rehaussement des digues allant jusqu'à $2 \mathrm{~m}$ par rapport à l'état actuel sur $1500 \mathrm{ml}$ le long de la zone urbaine. Rive droite, les modélisations de l'état aménagé permettent de préciser les zones de submersion, dont la surface est estimée à 148 ha (aléa fort) et les secteurs d'aménagement possibles. Un état de l'art des aménagements réalisés dans ce type de contexte avec analyse multicritères et retour sur expérience va permettre d'élaborer des solutions adaptées en fonction des biens et des personnes à protéger.

\section{Références bibliographiques}

ABHS -Agence Du Bassin Hydraulique du Sebou- (2008). Crue du 10 octobre 2008 dans le Haut Sebou. Royaume du Maroc. Rapport technique.

DGEC -Direction Générale de l'Energie et du Climat / Service du climat et de l'efficacité énergétique / Observatoire national sur les effets du réchauffement climatique- (2010). Prise en compte de l'élévation du niveau de la mer en vue de l'estimation des impacts du changement climatique et des mesures d'adaptation possibles. Synthèse $\mathrm{n}^{\circ} 2 \mathrm{Fev} .2010$.

EL BLIDI S., FEKHAOUI M. (2003). Hydrologie et dynamique marégraphique de l'estuaire du Sebou (Gharb, Maroc). Bulletin de l'Institut Scientifique, Rabat, section Sciences de la Vie, $\mathrm{n}^{\circ} 25$, 57-65.

SOGREAH, LPEE (2011). Etude d'expertise sédimentologique de la plage de Mehdia. Direction des Ports et du Domaine Public. 